\title{
Impact of pathological response after preoperative transcatheter arterial chemoembolization (TACE) on incidences of microvascular invasion and early tumor recurrence in hepatocellular carcinoma: a multicenter propensity score matching analysis
}

\author{
Yun Yang $^{1 \#}$, Zheng Dang ${ }^{2 \#}$, Peng Lu ${ }^{3 \#}$, Youwen Qian ${ }^{4 \#}$, Kongying Lin ${ }^{5}$, Zeya Pan ${ }^{1}$, Wan Yee Lau ${ }^{1,6}$, \\ Weiping Zhou ${ }^{1,7,8}$
}

${ }^{1}$ The Third Department of Hepatic Surgery, Eastern Hepatobiliary Surgery Hospital, Shanghai, China; ${ }^{2}$ Department of Hepatobiliary Surgery, 940 Hospital of PLA Joint Logistic Support Force, Lanzhou, China; ${ }^{3}$ Department of Hepatobiliary Surgery, Hainan Hospital of Chinese PLA General Hospital, Sanya, China; ${ }^{4}$ Department of Pathology, Eastern Hepatobiliary Surgery Hospital, Shanghai, China; ${ }^{5}$ Department of Hepatopancreatobiliary Surgery, Mengchao Hepatobiliary Hospital of Fujian Medical University, Fuzhou, China; ${ }^{6}$ Faculty of Medicine, the Chinese University of Hong Kong, Hong Kong, China; ${ }^{7}$ Key Laboratory of Signaling Regulation and Targeting Therapy of Liver Cancer (SMMU), Ministry of Education, Shanghai, China; ${ }^{8}$ Shanghai Key Laboratory of Hepatobiliary Tumor Biology (EHBH), Shanghai, China

Contributions: (I) Conception and design: WY Lau, W Zhou; (II) Administrative support: W Zhou; (III) Provision of study materials or patients: Y Yang, Z Dang, P Lu, Y Qian; (IV) Collection and assembly of data: K Lin; (V) Data analysis and interpretation: Z Pan; (VI) Manuscript writing: All authors; (VII) Final approval of manuscript: All authors.

"These authors contribute equally to this work.

Correspondence to: Professor Wan Yee Lau, MD, FRCS, FRACS(Hon). Faculty of Medicine, the Chinese University of Hong Kong, Shatin, New Territories, Hong Kong, China. Email: josephlau@cuhk.edu.hk; Dr. Weiping Zhou, MD, PhD. The Third Department of Hepatic Surgery, Eastern Hepatobiliary Surgery Hospital, 225 Changhai Road, Shanghai 200438, China. Email: ehphwp@126.com.

Background: To study the influence of pathological responses (PR) after transcatheter arterial chemoembolization (TACE) on incidences of microvascular invasion (MVI) and early recurrence in hepatocellular carcinoma (HCC) patients.

Methods: Between 2013 to 2015, consecutive HCC patients who underwent liver resection with "curative" intent at three hospitals were enrolled in this study. Patients with different areas of PR after preoperative TACE were compared with those without preoperative TACE on the incidences of MVI, early recurrence rates and patterns of recurrence before and after propensity score matching (PSM).

Results: Of 1,970 patients, 737 patients who received preoperative TACE were divided into three groups according to the areas of PR: $\geq 90 \%$ ( $n=226), 60-90 \%$ ( $n=447$ ), and $<60 \%(n=64)$. PR $\geq 90 \%$ was an independent protective factor of incidences of MVI [odds ratio (OR), 0.144; $95 \%$ confidence interval (CI), $0.082-0.245, \mathrm{P}<0.001$ ) and early recurrence (HR, 0.742; 95\% CI, 0.561-0.963, $\mathrm{P}=0.032$ ); while $\mathrm{PR}<60 \%$ was an independent risk factor of incidences of MVI (OR, 6.076; 95\% CI, 3.004-11.728, $\mathrm{P}<0.001)$ and early recurrence (HR, 1.428; 95\% CI, 1.095-1.929; P=0.009). Furthermore, patients with $\mathrm{PR}<60 \%$ were significantly more likely to develop multiple intrahepatic recurrences involving multiple hepatic segments when compared with patients without preoperative TACE.

Conclusions: This study indicated the area of PR after TACE was closely associated with the incidences of MVI and early tumor recurrence. Patients with PR $<60 \%$ were at significantly higher risks of having more MVI, early and multiple tumor recurrences

Keywords: Hepatocellular carcinoma (HCC); transcatheter arterial chemoembolization (TACE); pathological response; microvascular invasion (MVI); early recurrence 
Submitted Sep 07, 2020. Accepted for publication Nov 25, 2020.

doi: $10.21037 / \mathrm{hbsn}-20-700$

View this article at: http://dx.doi.org/10.21037/hbsn-20-700

\section{Introduction}

Hepatocellular carcinoma (HCC) is a common malignant tumor worldwide (1). Liver resection remains the mainstay of curative treatment for eligible HCC patients. Although surgical treatments of HCC have made tremendous progress in recent years, the prognosis of HCC is still unsatisfactory because of high tumor recurrence rates $(2,3)$, which remain as great challenges to surgeons.

The interval between the date of surgery and tumor recurrence has been used to divide HCC recurrence into early ( $<2$ years) and late recurrences ( $\geq 2$ years) (4). More than $60 \%$ of all HCC recurrences are early recurrences closely related to presence of microvascular invasion (MVI) (5-9). Previous studies have shown that approximately 20$60 \%$ of HCC specimens obtained after liver resection were accompanied by MVI (10), which can be found in early and even very early stages of HCC (11).

To our knowledge, the impact of pathological responses (PR) of HCC after preoperative transcatheter arterial chemoembolization (TACE) on the incidences of MVI and the patterns of early recurrence have not been studied. A large multicenter study was conducted to study the association of PR after preoperative TACE on the incidences of MVI and early tumor recurrence in patients who underwent partial hepatectomy to treat resectable HCC with curative intent. Propensity score matching (PSM) was used to reduce potential biases which are inherent in retrospective studies. We present the following article in accordance with the STROBE reporting checklist (available at https://hbsn.amegroups.com/article/view/10.21037/ hbsn-20-700/rc).

\section{Methods}

\section{Study population}

Between January 1, 2013 and December 1, 2015, consecutive HCC patients who underwent R0 resection at three hospitals in China (Eastern Hepatobiliary Surgery Hospital, 940 Hospital of PLA Joint Logistic Support Force, and Hainan Hospital of Chinese PLA General Hospital) were assessed to enter into this study. A preoperative diagnosis of $\mathrm{HCC}$ was based on the diagnostic criteria used by the American Association for the Study of Liver Diseases (12). The inclusion and exclusion criteria for this study are listed in Table S1 and Figure S1. The present study was performed in accordance with the Declaration of Helsinki (as revised in 2013). The study was approved by the Ethics Committees of all the study centers (No. EHBHKY2020-K-056), and written informed consent was obtained from all participants for their data to be used in clinical research.

\section{Preoperative evaluations}

Routine preoperative examinations included imaging and serological examinations. All patients underwent abdominal ultrasonography, intravenous contrast-enhanced abdominal magnetic resonance imaging (MRI) and/or computed tomography (CT) scans, and chest X-ray or chest CT scans. All radiological data was reviewed using a unified diagnostic criterion by two independent radiologists who had $>10$ years of radiological experience. Preoperative laboratory tests included routine blood tests, liver function, renal function, coagulation profile, hepatitis B surface antigen/ e antigen (HBsAg, HBeAg), hepatitis $\mathrm{C}$ virus antibody (HCV Ab), hepatitis B virus DNA load (HBV DNA), and levels of alpha-fetoprotein (AFP), carcinoembryonic antigen (CEA), and carbohydrate antigen 19-9 (CA199). Esophageal and gastric varices were diagnosed by esophagogastroduodenoscopy.

\section{Preoperative TACE}

TACE was performed as previously described (13). Briefly, a vascular catheter was inserted into a femoral artery using the Seldinger technique. The catheter tip was selectively inserted into the left or right hepatic artery, or the tumor-feeding artery when technically possible, and hepatic angiography was then performed. An emulsion of 5-fluorouracil (1 g), mitomycin C (20 mg), cisplatin (5 mg), and lipiodol (10-30 $\mathrm{mL}$ at $1-2 \mathrm{~mL} / \mathrm{cm}$ of tumor diameter) was then injected. This was followed by embolization with 1to 3-mm gelatin sponge particles until there was complete stasis in the tumor feeding vessels. In all cases, testing for a parasitic tumor blood supply was done through accessory 
arteries (i.e., the inferior phrenic, internal mammary, or intercostal arteries), and if one was present, the patient underwent additional superselective treatment (the same chemotherapeutic mixture plus embolization). The lobar technique was used in case of multiple ( $>3)$ HCC in the same lobe or when superselective cannulation of the feeding artery was not technically feasible.

When the operator was unable to treat all nodules in a single session, the first TACE was split into two sessions carried out within 1 month. All TACE procedures were performed by interventional physicians who had $>10$ years of interventional experience.

Routine blood tests, liver function, renal function, CEA, CA19-9, and AFP were carried out in all patients 4-6 weeks after TACE.

\section{Surgical procedures}

Resectability of HCC was evaluated based on patient's general condition, liver function, tumor size, tumor location, and amount of future remnant liver volume as estimated by CT and/or MRI scans before operation. The technique used for R0 liver resection had previously been reported (14). All liver surgery was performed by a hepatic surgeon with more than 20 years of experience.

\section{Postoperative histopathological examinations}

Surgical specimens were examined to assess tumor number, maximum tumor diameter, tumor margins, tumor encapsulation, satellite nodules, and liver cirrhosis. Satellite nodules were defined as tumors $<1 \mathrm{~cm}$ in diameter and located $<1 \mathrm{~cm}$ from the main tumor. All specimens were cut into slices along the longest diameter of the tumor at a thickness of $5 \mathrm{~mm}$. PR was determined as the percentage of the lesion with non-viable cancer cells (represented by necrosis or fibrosis, the ultimate stage of necrosis). When there were multiple tumors, the mean percentage was taken. Currently, the definition of MVI is presence of tumor cells in a portal vein, hepatic vein, or large capsular vessel of the surrounding hepatic tissue lined by endothelium that is only visible under microscopy (15). All histopathological evaluations were performed independently by two pathologists with $>10$ years of experience. The two pathologists were blinded to all clinical data. All discrepancies between pathologists were settled by discussion.

\section{Follow-up and endpoints}

All patients were reviewed once every 3 months after surgery. Postoperative follow-up was performed by the same team of surgeons, and the follow-up program included serum AFP, complete blood counts, liver function, renal function, hepatitis B virus DNA, chest X-ray, abdominal ultrasound, and intravenous contrast-enhanced abdominal CT or MRI. When a patient was diagnosed with tumor recurrence, appropriate treatments, including percutaneous ethanol injection, radiofrequency ablation, TACE, or re-hepatectomy were performed based on the patient's general condition, liver function, tumor size, pattern of tumor recurrence, as well as patient's wishes. Treatment of extrahepatic metastasis included local excision, systemic chemotherapy, and molecular targeted therapy. Best supportive care was given to patients with end-stage HCC, poor liver function, or poor general status. This study was censored on Jun 1, 2019.

The median (interquartile range) duration of follow-up was $31.3(20.5-60.2)$ months. The primary endpoint was time to recurrence, which was calculated from the date of surgery to the date when tumor recurrence was diagnosed. The secondary endpoint was presence of MVI based on postoperative histopathology.

\section{PSM}

PSM was used to eliminate selection biases produced by disequilibrium between groups according to previous descriptions $(16,17)$. Covariates which were entered into the PSM model included age, sex, HbsAg, HbeAg, HCV Ab, HBV-DNA load, AFP levels, total bilirubin (TBIL), albumin (ALB), alanine aminotransferase (ALT), preoperative platelets (PLT) count, tumor number, cirrhosis, maximum tumor size, tumor encapsulation, tumor margin, tumor differentiation, anatomical resection, surgical margin, and satellite nodules. PSM was performed as a 1:1 matching between the two groups, with nearest-neighbor matching and a 0.05 caliper width using SPSS. The matching procedure was based on a previous report (18).

\section{Statistical analysis}

Continuous variables were expressed as median (range) or mean [standard deviation (SD)]. Categorical variables were expressed as frequency (percentage). Categorical 


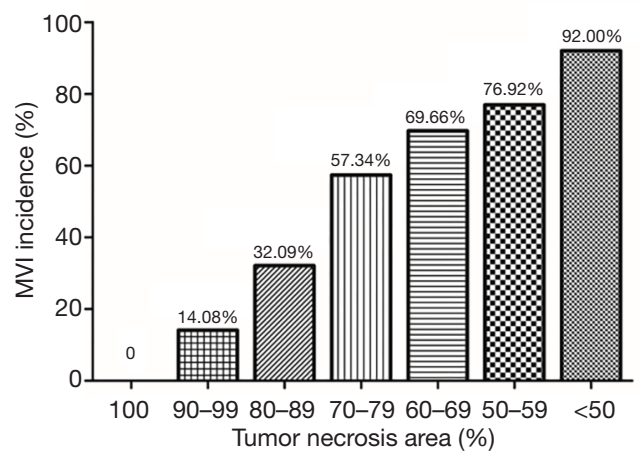

Figure 1 Association of incidence of MVI with tumor PR area. MVI microvascular invasion; PR, pathological response.

variables were compared by the $\chi^{2}$ test or Fisher's exact test. Continuous variables were compared by the Student's $t$-test or Mann-Whitney U test. All analyses were two-tailed. Survival curves were calculated using the Kaplan-Meier method and compared using the log-rank test. Preoperative factors that might be associated with MVI were assessed by univariable and multivariable logistic regression analyses. Cox regression analyses were used for univariable and multivariable analyses of early recurrence. All statistical analyses were performed using SPSS 23.0 for Windows (SPSS, IBM, Armonk, NY, USA). A P value $<0.05$ was considered statistically significant.

\section{Results}

\section{Baseline patient clinical characteristics}

Of 3,093 patients who underwent $\mathrm{R} 0$ resection, 1,123 patients were excluded from this study based on the exclusion criteria as listed in Table S1 and Figure S1. Finally, data on the remaining 1,970 patients were analyzed.

Of 1,970 patients, 737 received, while the remaining 1,233 did not receive, preoperative TACE. The reasons why these 737 patients received TACE as the first treatment were because of personal/medical reasons to delay major surgery $(n=293)$, initial refusal to surgical treatment $(n=297)$ or socio-financial reasons ( $\mathrm{n}=147)$. Among the 737 patients who were treated with preoperative TACE, 543 underwent a single session of preoperative TACE and 194 multiple sessions. The median number of preoperative TACE sessions was 1 (range, 1-6). The median interval between the first TACE treatment and surgery was 6 weeks (range, 4-44 weeks). For patients with multiple preoperative TACE sessions, the median interval between the last TACE treatment and surgery was 8 weeks (range, 4-46 weeks).

Baseline clinical characteristics of the patients with and without preoperative TACE are shown in Table S2. Differences in age, percentages of $\mathrm{HBeAg}(+), \mathrm{HCV}$ $\mathrm{Ab}(+)$, and tumor differentiation between groups were not significant $(\mathrm{P}>0.05)$. However, there were significant differences between groups in serum AFP, TBIL and ALT levels, tumor size, gender, HBsAg positivity rates, associated liver cirrhosis, HBV DNA loads, ALB and PLT levels and percentages of patients with smooth tumor margins, multiple tumors, complete tumor capsules and satellite lesions (all $\mathrm{P}<0.05$ ). In addition, the proportions of patients who received antivirus treatment, anatomical resection and wide surgical margins were also significantly different (all $\mathrm{P}<0.05)$. After PSM, 602 pairs of patients were created. The background characteristics and preoperative factors between groups became similar (all $\mathrm{P}>0.05$ ) (Table S2).

\section{Association of PR with incidences of MVI}

The association between preoperative TACE with incidences of MVI based on tumor PR was analyzed in the 737 patients who underwent preoperative TACE. As shown in Figure 1, the incidence of MVI in patients with complete pathological response (CPR) was $0(0 / 84)$. The incidences of MVI gradually increased with decreasing percentages of tumor PR. In patients with a tumor PR area $<50 \%$, the incidence of MVI was as high as $92.0 \%$. The incidence of MVI in the group of patients with preoperative TACE was significantly lower than those without preoperative TACE before PSM (38.8\% vs. $44.7 \%, \mathrm{P}=0.011)$. After PSM, the difference was not significant $(37.5 \%$ vs. $40.9 \%, \mathrm{P}=0.238)$ (Table 1). The 737 patients who underwent preoperative TACE were then divided into three groups according to tumor PR: $\geq 90 \%(n=226), 60-90 \%(n=447)$, and $<60 \%$ $(n=64)$. The clinical characteristics of the patients with a tumor $\mathrm{PR} \geq 90 \%$ and patients without preoperative TACE before and after PSM are shown in Table S3. The incidence of MVI in the group of patients with a tumor PR $\geq 90 \%$ was $8.8 \%$, which was significantly lower than those patients without preoperative TACE before PSM $(8.8 \% v s$. $44.7 \%, \mathrm{P}<0.001)$, and after PSM ( $8.1 \%$ vs. $34.2 \%, \mathrm{P}<0.001)$ (Table 1). The pathology images of different PR areas with or without MVI are shown in Figure S2.

The clinical characteristics of patients with a tumor PR of $60-90 \%$ and patients without preoperative TACE before and after PSM are shown in Table S4. The incidence of MVI in patients with a tumor PR of $60-90 \%$ was similar to 
Table 1 MVI incidences between patients with different PR and without preoperative TACE before and after PSM

\begin{tabular}{|c|c|c|c|c|c|c|}
\hline \multirow{2}{*}{ Group } & \multicolumn{6}{|c|}{ MVI incidence, n (\%) } \\
\hline & $\begin{array}{l}\text { With preoperative } \\
\text { TACE }(\mathrm{N}=737)\end{array}$ & $\begin{array}{l}\text { Without preoperative } \\
\text { TACE }(\mathrm{N}=1,233)\end{array}$ & $\mathrm{P}$ & $\begin{array}{l}\text { With preoperative } \\
\text { TACE }(\mathrm{N}=602)\end{array}$ & $\begin{array}{l}\text { Without preoperative } \\
\text { TACE }(\mathrm{N}=602)\end{array}$ & $\mathrm{P}$ \\
\hline Total & 286/737 (38.8) & $551 / 1,233(44.7)$ & 0.011 & 226/602 (37.5) & 246/602 (40.9) & 0.238 \\
\hline With PR area $<60 \%$ & $53 / 64(82.8)$ & $551 / 1,233(44.7)$ & $<0.001$ & 44/50 (88.0) & $24 / 50(48.0)$ & $<0.001$ \\
\hline
\end{tabular}

MVI, microvascular invasion; PR, pathological responses; TACE, transarterial chemoembolization; PSM, propensity score matching.

patients without preoperative TACE either before or after PSM (before PSM: $47.7 \%$ vs. $44.7 \%, \mathrm{P}=0.281$; after PSM: $47.1 \%$ vs. $43.3 \%, \mathrm{P}=0.268$ ) (Table 1 ).

The clinical characteristics of patients with a tumor PR $<60 \%$ and patients without preoperative TACE before and after PSM are shown in Table S5. The incidence of MVI in the group of patients with a tumor $\mathrm{PR}<60 \%$ was $82.8 \%$, which was significantly higher than those patients without preoperative TACE before PSM (82.8\% vs. 44.7\%, $\mathrm{P}<0.001)$. After $\mathrm{PSM}$, this was $88.0 \%$, which remained significantly higher than those patients without preoperative TACE $(88.0 \%$ vs. $48.0 \%, \mathrm{P}<0.001)$ (Table 1$)$.

Further analyses were conducted on the entire patient cohort $(\mathrm{n}=1,970)$. The results of univariable analysis are shown in Table S6. Multivariable analysis indicated that large tumor size, satellite nodules, grade III/IV differentiation, non-smooth tumor margins, and high pre-treatment AFP levels were independently associated with increased risks of MVI. However, anti-virus treatment was an independent protective factor of MVI. In this entire cohort, preoperative TACE and the number of TACE sessions had no impact on the incidence of MVI. PR $\geq 90 \%$ was an independent protective factor of MVI [odds ratio (OR), 0.144; $95 \%$ confidence interval (CI), 0.082-0.245, $\mathrm{P}<0.001]$, however, $\mathrm{PR}<60 \%$ was an independent risk factor of MVI (OR, 6.076; 95\% CI, 3.004-11.728, $\mathrm{P}<0.001$ ) (Table 2).

\section{Association of PR with early tumor recurrence}

The 6-, 12-, and 24-month tumor recurrence rates in patients with tumor $\mathrm{PR} \geq 90 \%$ were significantly lower than those patients without preoperative TACE before and after PSM (both $\mathrm{P}<0.001$ before and after PSM)
(Figure $2 A, 2 B)$. In patients with tumor $\mathrm{PR}$ between $60-90 \%$, The 6-, 12-, and 24-month tumor recurrence rates were comparable between patients with tumor PR between 60-90\% and patients without preoperative TACE before and after PSM $(\mathrm{P}=0.183$ before $\mathrm{PSM}$, and $\mathrm{P}=0.364$ after $\mathrm{PSM})$ (Figure $2 C, 2 D$ ). In patients with tumor $\mathrm{PR}<60 \%$, the 6-, $12-$, and 24-month tumor recurrence rates were significantly higher than those patients without preoperative TACE before and after PSM $(\mathrm{P}<0.001$ before PSM, and $\mathrm{P}=0.042$ after PSM) (Figure 2E,2F).

Independent risk factors for early tumor recurrence were identified using cox regression analyses on the entire cohort $(\mathrm{n}=1,970)$. The results of univariable analysis are shown in Table S7. Multivariable analysis indicated that tumor size $\geq 5 \mathrm{~cm}$ [hazard ratio (HR), 1.765; 95\% CI, 1.528-2.016; $\mathrm{P}<0.001]$, AFP levels $\geq 400 \mathrm{ng} / \mathrm{mL}$ (HR, 1.244; 95\% CI, 1.077-1.426; $\mathrm{P}=0.003)$, and presence of MVI (HR, 1.696; 95\% CI, 1.508-2.009; $\mathrm{P}<0.001)$ were independently associated with early tumor recurrence (Table 3) In contrast, anti-virus treatment was an independent protective factor for early tumor recurrence (HR, 0.792; 95\% CI, 0.611-0.978; $\mathrm{P}=0.035)$. Interestingly, cox regression analyses on this entire cohort revealed that preoperative TACE and the number of preoperative TACE sessions had no impact on early tumor recurrence. However, tumor PR area after TACE was a significant factor associated with early tumor recurrence. Tumor PR $\geq 90 \%$ was an independent protective factor for early tumor recurrence (HR, 0.742; 95\% CI, 0.561-0.963; $\mathrm{P}=0.032$ ), while tumor $\mathrm{PR}<60 \%$ was an independent risk factor for early tumor recurrence (HR, 1.428; $95 \%$ CI, 1.095-1.929; $\mathrm{P}=0.009$ ) (Table 3); and tumor PR between 60$90 \%$ had no impact on early tumor recurrence (HR, 1.095; 95\% CI, 0.930-1.289; $\mathrm{P}=0.276$ ) (Table S7). 
Table 2 Multivariable logistic regression analysis of microvascular invasion presence in patients receiving preoperative TACE treatment and those not receiving preoperative TACE treatment $(\mathrm{N}=1,970)$

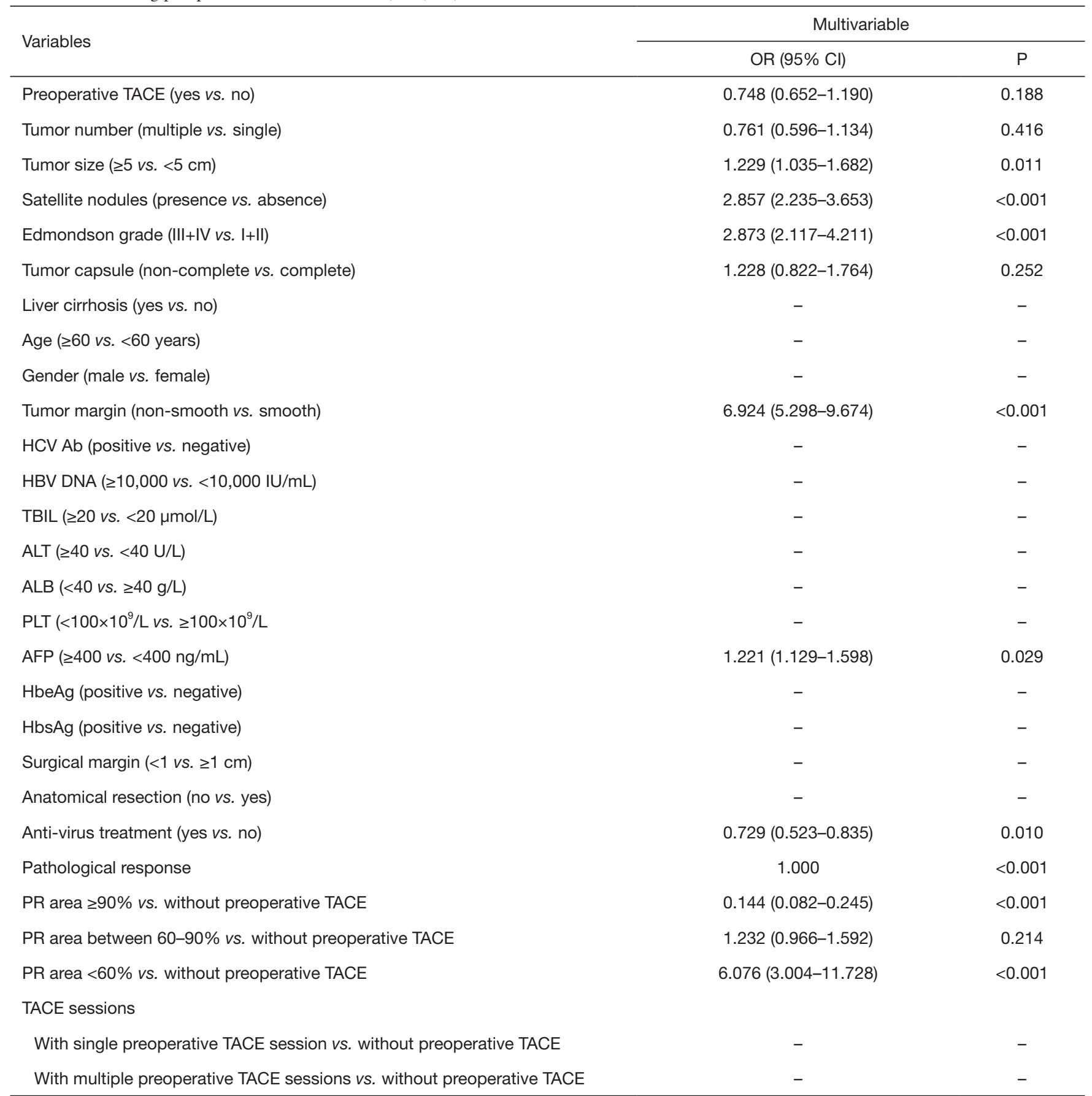

TACE, transcatheter arterial chemoembolization; 95\% Cl, 95\% confidence interval; OR, odds ratio; HCV Ab, hepatitis C virus antibody; HBV, hepatitis B virus; DNA, deoxyribonucleic acid; TBIL, total bilirubin; ALT, alanine aminotransferase; ALB, albumin; PLT, platelet; AFP, serum alpha-fetoprotein; $\mathrm{HBeAg}$, hepatitis $\mathrm{B}$ e antigen; HBsAg, hepatitis $\mathrm{B}$ surface antigen; PR, pathological response. 


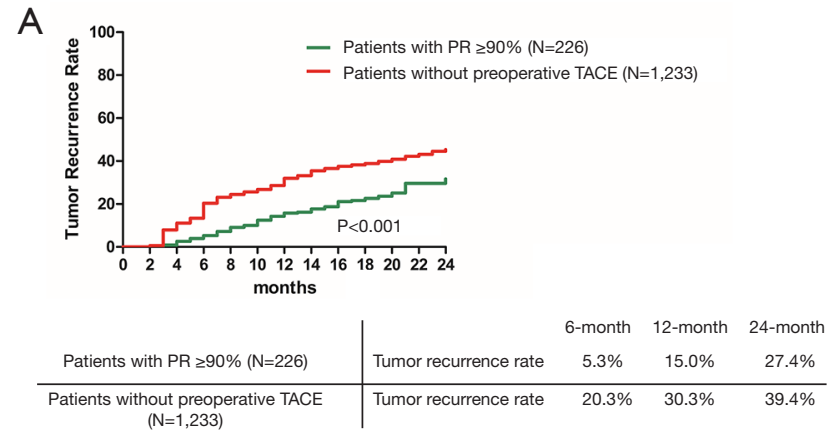

C

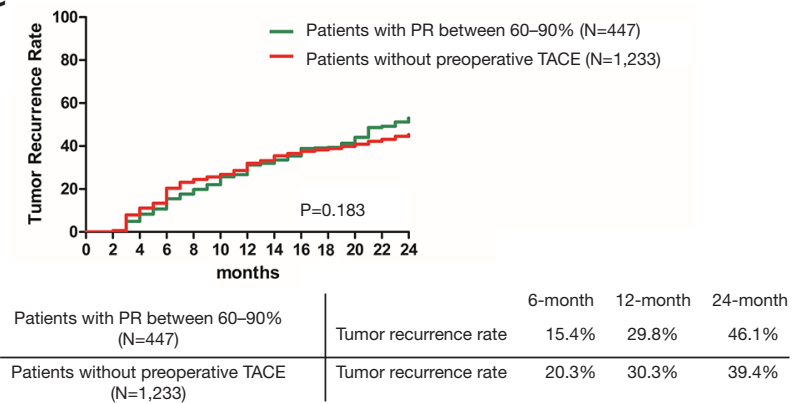

$\mathrm{E}$

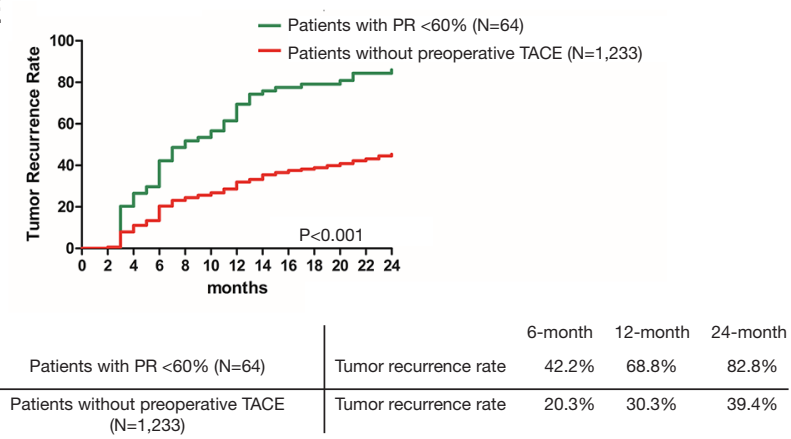

B

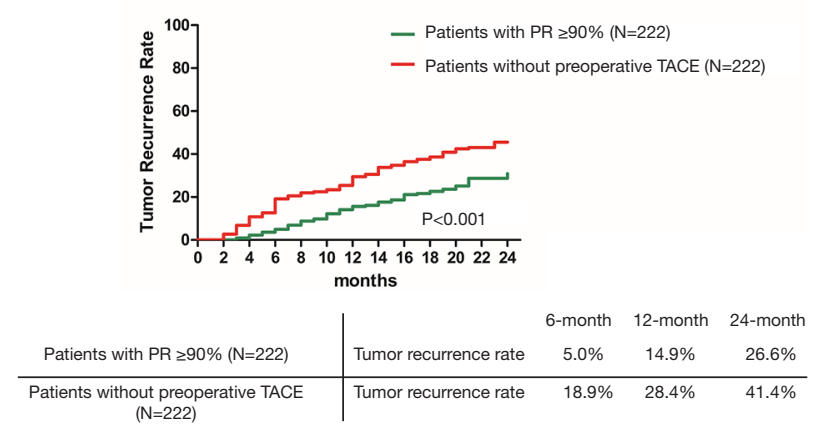

$\mathrm{D}$

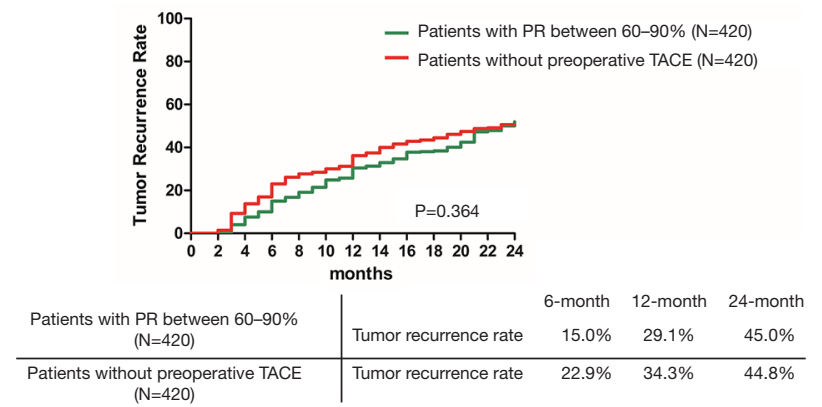

$\mathrm{F}$

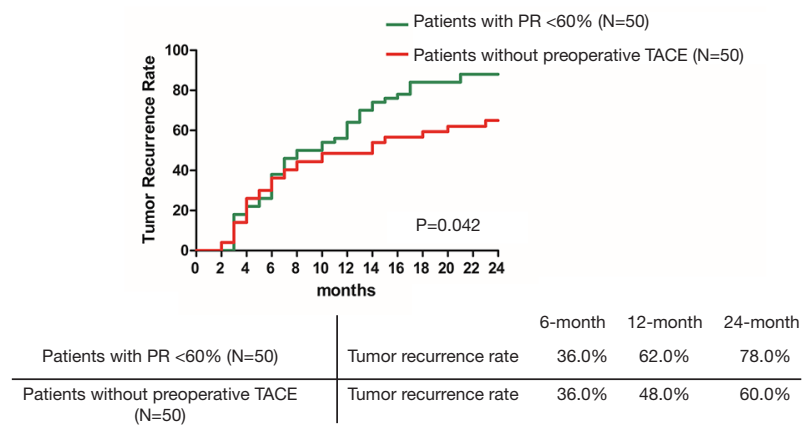

Figure 2 Early tumor recurrence between patients with different tumor PR and patients without preoperative TACE. (A) The cumulative early tumor recurrence curve of patients with tumor $\mathrm{PR}$ area $\geq 90 \%$ and patients without preoperative TACE before $\mathrm{PSM}(\mathrm{P}<0.001)$. (B) The cumulative early tumor recurrence curve of patients with tumor PR area $\geq 90 \%$ and patients without preoperative TACE after PSM (P<0.001). (C) The cumulative early tumor recurrence curve of patients with tumor PR area between 60-90\% and patients without preoperative TACE before PSM ( $\mathrm{P}=0.183$ ). (D) The cumulative early tumor recurrence curve of patients with tumor PR area between $60-90 \%$ and patients without preoperative TACE after PSM $(\mathrm{P}=0.364)$. (E) The cumulative early tumor recurrence curve of patients with tumor $\mathrm{PR}$ area $<60 \%$ and patients without preoperative TACE before PSM $(\mathrm{P}<0.001)$. (F) The cumulative early tumor recurrence curve of patients with tumor PR area $<60 \%$ and patients without preoperative TACE after PSM $(\mathrm{P}=0.042)$. PR, pathological response; TACE, transcatheter arterial chemoembolization; PSM, propensity score matching.

\section{Association of PR with patterns of early tumor recurrence}

The patterns of tumor recurrence have been described in a previous report (19). The type of recurrence in patients with $\mathrm{PR} \geq 90 \%$ was similar to patients without preoperative TACE, either before or after PSM (before PSM: $\mathrm{P}=0.265$; after PSM: $\mathrm{P}=0.205)$. However, the proportion of multiple intrahepatic recurrence was significantly higher in patients without preoperative TACE either before or after PSM (before PSM: $15.3 \%$ vs. $28.6 \%, \mathrm{P}=0.030$; after PSM: $15.5 \%$ vs. $31.6 \%, \mathrm{P}=0.031)$. In addition, patients with tumor $\mathrm{PR} \geq 90 \%$ were less likely to have local recurrences or recurrences involving multiple liver segments either before or after PSM (before PSM: $\mathrm{P}=0.033$; after PSM: $\mathrm{P}=0.025$ ) 
Table 3 Multivariable cox regression analysis of early tumor recurrences $(\mathrm{N}=1,970)$

\begin{tabular}{|c|c|c|}
\hline Variables & \multicolumn{2}{|c|}{ Multivariable } \\
\hline Preoperative TACE (yes vs. no) & - & - \\
\hline Tumor number (multiple vs. single) & $1.252(1.126-1.596)$ & $<0.001$ \\
\hline Tumor size ( $\geq 5$ vs. $<5 \mathrm{~cm}$ ) & $1.765(1.528-2.016)$ & $<0.001$ \\
\hline Edmondson grade (III+IV vs. I+II) & $1.118(0.877-1.346)$ & 0.295 \\
\hline Tumor capsule (non-complete vs. complete) & $1.219(0.999-1.518)$ & 0.055 \\
\hline Liver cirrhosis (yes vs. no) & - & - \\
\hline Age ( $\geq 60$ vs. $<60$ years) & $1.064(0.897-1.169)$ & 0.427 \\
\hline HCV Ab (positive vs. negative) & - & - \\
\hline HBV DNA ( $\geq 10,000$ vs. $<10,000$ IU/mL) & - & - \\
\hline TBIL ( $\geq 20$ vs. $<20 \mu \mathrm{mol} / \mathrm{L})$ & - & - \\
\hline ALT ( $\geq 40$ vs. $<40 \mathrm{U} / \mathrm{L})$ & - & - \\
\hline ALB (<40 vs. $\geq 40 \mathrm{~g} / \mathrm{L})$ & - & - \\
\hline PLT $\left(<100 \times 10^{9} /\right.$ L vs. $\geq 100 \times 10^{9} / \mathrm{L}$ & - & - \\
\hline $\operatorname{AFP}(\geq 400$ vs. $<400 \mathrm{ng} / \mathrm{mL})$ & $1.244(1.077-1.426)$ & 0.003 \\
\hline HbeAg (positive vs. negative) & - & - \\
\hline PR area $\geq 90 \%$ vs. without preoperative TACE & 1 & \\
\hline PR area between $60-90 \%$ vs. without preoperative TACE & $0.742(0.561-0.963)$ & 0.032 \\
\hline PR area $<60 \%$ vs. without preoperative TACE & $1.036(0.869-1.119)$ & 0.812 \\
\hline TACE sessions & $1.428(1.095-1.929)$ & 0.009 \\
\hline \multicolumn{3}{|l|}{ With single preoperative TACE session vs. without preoperative TACE } \\
\hline With multiple preoperative TACE sessions vs. without preoperative TACE & - & - \\
\hline Preoperative TACE (yes vs. no) & - & - \\
\hline
\end{tabular}

TACE, transcatheter arterial chemoembolization; $95 \% \mathrm{Cl}, 95 \%$ confidence interval; OR, odds ratio; HCV Ab, hepatitis $\mathrm{C}$ virus antibody; HBV, hepatitis B virus; DNA, deoxyribonucleic acid; TBIL, total bilirubin; ALT, alanine aminotransferase; ALB, albumin; PLT, platelet; AFP, serum alpha-fetoprotein; $\mathrm{HBeAg}$, hepatitis B e antigen; HBsAg, hepatitis B surface antigen; PR, pathological response. 


\section{(Figure S3).}

Patients with PR of $60-90 \%$ were more likely to have extrahepatic metastasis and multiple intrahepatic recurrences than patients without preoperative TACE before PSM (14.6\% vs. $6.4 \%, \mathrm{P}=0.001 ; 38.1 \%$ vs. $28.6 \%$, $\mathrm{P}=0.019$, respectively). However, the differences between groups became insignificant after PSM (9.2\% vs. $11.7 \%$, $\mathrm{P}=0.417 ; 37.9 \%$ vs. $33.7 \%, \mathrm{P}=0.427$, respectively). In addition, the differences in locations of intrahepatic recurrences between groups were also insignificant either before or after PSM (before PSM: $\mathrm{P}=0.684$; after PSM: $\mathrm{P}=0.630$ ) (Figure $\mathrm{S} 3$ ).

Extrahepatic metastases were more common in patients with $\mathrm{PR}<60 \%$ than in patients without preoperative TACE before PSM $(\mathrm{P}<0.001)$. After PSM, the proportions of patients developing extrahepatic metastases were similar between groups $(\mathrm{P}=0.726)$. Patients with $\mathrm{PR}<60 \%$ were more likely to have multiple intrahepatic tumor recurrences either before or after PSM (before PSM: $\mathrm{P}=0.001$; after PSM: $\mathrm{P}=0.029)$. In addition, local recurrence or recurrent tumors which involved multiple liver segments were more commonly seen in patients with $\mathrm{PR}<60 \%$, either before or after PSM (before PSM: $\mathrm{P}=0.041$; after PSM: $\mathrm{P}=0.024$ ) (Figure S3).

\section{Association of PR with overall survival (OS)}

The 1-, 3-, and 5-year cumulative OS rates in patients with tumor PR $\geq 90 \%$ were significantly higher than patients without preoperative TACE before and after PSM. $(\mathrm{P}<0.001$ before $\mathrm{PSM}$, and $\mathrm{P}=0.014$ after $\mathrm{PSM}$ ) (Figure $3 A, B$ ). For patients with tumor PR between $60-90 \%$, the 1-, 3-, and 5 -year cumulative OS rates were similar with patients without preoperative TACE before and after PSM $(\mathrm{P}=0.259$ before $\mathrm{PSM}$, and $\mathrm{P}=0.604$ after PSM) (Figure 3C,D). The 1-, 3 -, and 5-year cumulative OS rates of patients with tumor $\mathrm{PR}<60 \%$ were significantly lower than patients without preoperative TACE before and after PSM $(\mathrm{P}<0.001$ before $\mathrm{PSM}$, and $\mathrm{P}=0.045$ after $\mathrm{PSM}$ ) (Figure $3 E, F)$.

\section{Discussion}

Approximately 20-60\% of HCC specimens are reported to be accompanied with MVI after liver resection, and MVI is a known high-risk factor that leads to early recurrence after curative liver resection for $\operatorname{HCC}(10,15,20,21)$. The aim of this study was to evaluate the influence of PR after TACE on incidences of MVI and prognosis in HCC patients.
Previous studies have reported on several models which are useful in predicting presence of MVI before liver resection $(22,23)$. By using these models, patients predicted to have high risks of MVI presence can be offered preoperative TACE to reduce the incidence of MVI, and hopefully prolong survival $(22,23)$.

Preoperative TACE with $\mathrm{PR} \geq 90 \%$ was associated with lower MVI incidence and better prognosis for HCC patients in this study. A single tumor and tumor size $\leq 5 \mathrm{~cm}$ have been reported to associate significantly with tumor PR $>90 \%(24,25)$. In addition, avid tumor enhancement, $\mathrm{AFP}<100 \mathrm{ng} / \mathrm{mL}$, and a longer time between the first TACE procedure and liver resection were all related to PR $>90 \%$ (26,27). Furthermore, development of $>90 \%$ lesion necrosis was reported to be due to a relatively simple blood supply to HCC and presence of a feeding vessel larger than $0.9 \mathrm{~mm}$ in diameter on pre-TACE visceral angiogram $(26,28)$. Golfieri et al. suggested that complete necrosis and necrosis $\geq 90 \%$ were more frequently observed after selective/superselective TACE than lobar TACE (29). However, selective/superselective TACE needs more time and skills. In this study, superselective TACE was carried out whenever technically feasible. Previous studies also indicated that TACE mainly caused necrosis of welldifferentiated HCC cells, leaving poorly differentiated cells which are less sensitive to hypoxia. Thus, tumors with PR $>90 \%$ are often homogeneous and well differentiated $(30,31)$. Currently, there is still no consensus on technique, protocol or drug regimen used in TACE. The impacts of dosage of chemo-agents and lipiodol, and size of gelatin sponges on tumor PR after preoperative TACE are unclear and need to be further studied. In this study, the dosage of chemo-agents and lipiodol, and size of gelatin sponges were dependent on tumor size, tumor number, tumor location, vascular condition of tumor feeding artery, and patients' liver function with or without accompanying liver cirrhosis.

The impact of bridging treatments on MVI in transplant candidates have been investigated (32). The study indicated that the incidence of MVI in patients with tumor necrosis $>60 \%$ was significantly higher than patients with tumor necrosis $0-60 \%$. Thus, the incidence of MVI was closely associated with the area of tumor necrosis, which was consistent with our findings. Results from multivariable analysis in our study indicated that not preoperative TACE, but different tumor PR areas after preoperative TACE led to different incidences of MVI. There is still no direct evidence to indicate incomplete TACE induces MVI. However, some experiments have shown that serum vascular endothelial 

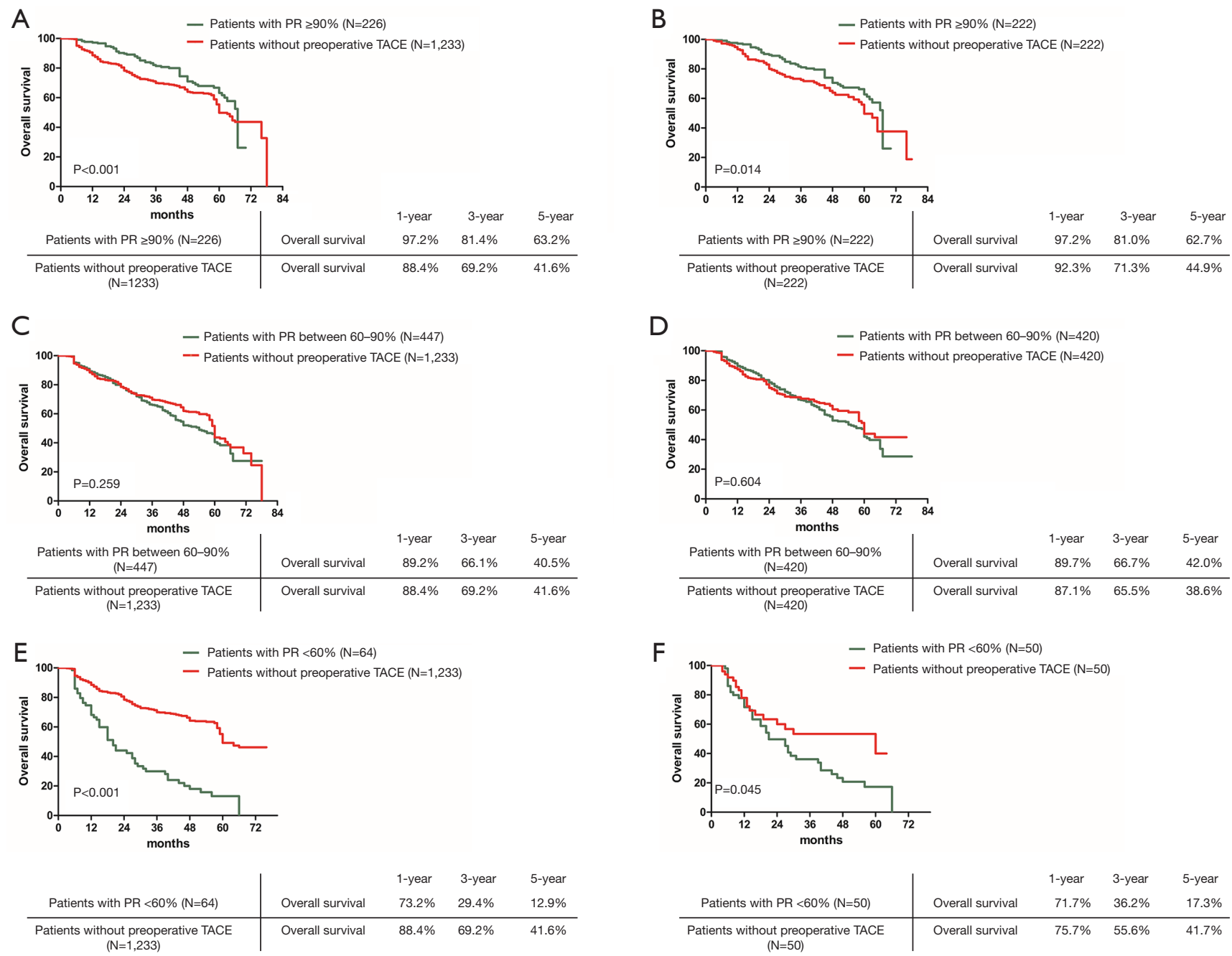

Figure 3 OS between patients with different tumor PR and patients without preoperative TACE. (A) The cumulative OS curve of patients with tumor PR area $\geq 90 \%$ and patients without preoperative TACE before PSM $(\mathrm{P}<0.001)$. (B) The cumulative OS curve of patients with tumor PR area $\geq 90 \%$ and patients without preoperative TACE after PSM ( $\mathrm{P}=0.014)$. (C) The cumulative OS curve of patients with tumor $\mathrm{PR}$ area between 60-90\% and patients without preoperative TACE before PSM $(\mathrm{P}=0.259)$. (D) The cumulative OS curve of patients with tumor PR area between 60-90\% and patients without preoperative TACE after PSM $(\mathrm{P}=0.604)$. (E) The cumulative OS curve of patients with tumor PR area $<60 \%$ and patients without preoperative TACE before PSM $(\mathrm{P}<0.001)$. (F) The cumulative OS curve of patients with tumor PR area $<60 \%$ and patients without preoperative TACE after PSM ( $\mathrm{P}=0.045)$. OS, overall survival; PR, pathological response; TACE, transcatheter arterial chemoembolization; PSM, propensity score matching.

growth factor (VEGF) or hypoxia inducible factor-1 alpha $(\mathrm{HIF}-1 \alpha)$ increase in suboptimal responders when compared with complete responders after TACE (33-36). To adapt to hypoxic environment, HIF- $1 \alpha$ stimulates cyclooxygenase-2 (COX-2) protein expression and promotes epithelial-to-mesenchymal transition (EMT) process in residual HCC cells, with resultant aggravation of HCC invasion and metastasis after TACE. Furthermore, EMT has been reported to be associated with formation of MVI (37-39). Based on these reports, TACE can be speculated to kill well-differentiated HCC cells while leaving behind those HCC cells which exhibit more aggressive behaviors, such as tumors with MVI. Incomplete necrosis of HCC also increases the expressions of VEGF and HIF-1 $\alpha$, which induce COX-2 expression and promote EMT process in residual HCC cells. EMT can further promote formation 
of MVI and enhance HCC invasion and metastasis, to contribute to poor prognosis in HCC patients after TACE. Further studies are needed to verify these speculations.

Our analysis revealed the association of tumor PR areas with the cumulative early tumor recurrence rates. Complete response (CR) after TACE leading to significantly better prognosis than partial response (PR) and stable disease (SD) have been reported (40-42). However, the impact of the area of PR on the incidences of MVI and early recurrence have not been reported. This study first reported that PR area $\geq 90 \%$ was an independent protective factor of incidence of MVI and early recurrence, and $\mathrm{PR}$ area $<60 \%$ was an independent risk factor of incidence of MVI and early recurrence. The incidences of MVI gradually increased with decreasing percentages of PR area. Furthermore, patients with $\mathrm{PR}<60 \%$ were significantly more likely to develop multiple intrahepatic recurrences with involvement of multiple hepatic segments when compared with patients without preoperative TACE. The influence of tumor PR on patterns of early tumor recurrence was again first reported in this study.

The tumor PR area was also found in this study to associate with the pattern of recurrence. Patients with a tumor PR area of $\geq 90 \%$ were less likely to have multiple intrahepatic, local or recurrences involving multiple liver segments than patients with a tumor PR area of $<60 \%$. Imaging features after TACE on CT and/or MRI were closely associated with $\mathrm{PR}$ area which had been validated in clinical practice. On post-TACE CT imaging, a lack of residual contrast enhancement, a decrease in lesion size, a high lesion density with accumulation of ethiodized oil, and a diffuse distribution of ethiodized oil throughout the lesion correlated with near-complete lesion necrosis upon histopathological analysis. In addition, semi-automated, three-dimensional (3D) quantitative assessment of HCC lesions with MRI after TACE showed a high degree of overall accuracy in prediction of histopathologic tumor necrosis. Enhancement-based and apparent diffusion coefficient-based MRI techniques are also useful in predicting pathologic tumor necrosis. Volumetric preTACE enhancement on MRI and post-TACE oil deposition in CT can accurately predict necrosis in TACE-treated HCC lesions. Thus, based on imaging features, the PR area could be predicted before liver resection $(26,43,44)$. For patients who were likely to have a tumor PR area of $<60 \%$ as predicted by CT and/or MRI, a wide surgical margin should be carried out to reduce the local recurrence rate (14). Although whether the width of surgical margin (narrow vs. wide margin) in liver resection for HCC patients affects long-term survival is still debated, the OS rate for patients with a wide margin $(>1 \mathrm{~cm})$ has been found to be significantly better than those with a narrow margin $(<1 \mathrm{~cm})$. In addition, all recurrence at the liver transection margin happened in the narrow margin group who had significantly worse survival outcomes than those without local recurrence (14). For patients with MVI, a resection margin of $>1 \mathrm{~cm}$ resulted in better 5 -year recurrence and overall survival than a resection margin of $<1 \mathrm{~cm}$. However, the differences in patients without MVI were not significant (45). Thus, for patients who are predicted to have a high risk of MVI by the established models, a widemargin liver resection should be carried out based on safety and technical feasibility. As patients with $\mathrm{PR}$ of $<60 \%$ were shown in this study to have poorer long-term survival outcomes after liver resection, these patients should be closely followed-up, and post-operative adjuvant treatment using TACE or molecular targeted agents should be discussed with these patients.

This study had several limitations. First, this is a retrospective study with potential biases inherent in such a type of study which can be reduced using PSM. However, PSM has limitations and it may even increase imbalance, inefficiency and biases between groups. After PSM, with 1:1 matching in this study, nearly half of the sample $(602 / 1,233)$ was reduced in patients without preoperative TACE, and thus resulting in a decrease in statistical power. Matching using 1:4 in PSM can lead to a minimum mean square error. As the sample size of patients without preoperation TACE in this study was twice of patients with preoperative TACE, 1:1 match was used. Furthermore, PSM only controls the influence of measurable variables. The way to resolve selection on unobservable is to enroll as many relevant measurable variables as possible (46). In this study, 21 variables associated with presence of MVI and prognosis were enrolled in PSM which can partly improve the statistical power. Second, the vast majority of HCC patients enrolled in this study had HBV infection because of the high prevalence of $\mathrm{HBV}$ infection in China. Patients with $\mathrm{HCV}$-related HCC and those with other etiologies will need to be further investigated to support the findings of this study.

In conclusion, PR area after TACE was shown in this study to be closely associated with presence of MVI, early tumor recurrences and patterns of recurrence. Measures to reduce incidences of MVI and to improve prognosis of patients after liver resection need further studies. 


\section{Acknowledgments}

Funding: This work was supported by grants from the National Natural Science Foundation of China (No. 81502416), the Science Fund for Creative Research Groups, NSFC, China, (No. 81521091), and the State Key Infection Disease Project of China (No. 2018ZX10732202-002-005).

\section{Footnote}

Reporting Checklist: The authors have completed the STROBE reporting checklist. Available at https://hbsn. amegroups.com/article/view/10.21037/hbsn-20-700/rc

Data Sharing Statement: Available at https://hbsn. amegroups.com/article/view/10.21037/hbsn-20-700/dss

Conflicts of Interest: All authors have completed the ICMJE uniform disclosure form (available at https://hbsn. amegroups.com/article/view/10.21037/hbsn-20-700/coif). WYL serves as an unpaid advisor of Hepatobiliary Surgery and Nutrition. The other authors have no conflicts of interest to declare.

Ethical Statement: The authors are accountable for all aspects of the work in ensuring that questions related to the accuracy or integrity of any part of the work are appropriately investigated and resolved. The present study was performed in accordance with the Declaration of Helsinki (as revised in 2013). The study was approved by the Institutional Ethics Committees of the Eastern Hepatobiliary Surgery Hospital, 940 Hospital of PLA Joint Logistic Support Force, and Hai Nan Hospital of Chinese PLA General Hospital (No. EHBHKY2020-K-056). Informed consent was obtained from all the patients for their data to be used for research.

Open Access Statement: This is an Open Access article distributed in accordance with the Creative Commons Attribution-NonCommercial-NoDerivs 4.0 International License (CC BY-NC-ND 4.0), which permits the noncommercial replication and distribution of the article with the strict proviso that no changes or edits are made and the original work is properly cited (including links to both the formal publication through the relevant DOI and the license). See: https://creativecommons.org/licenses/by-nc-nd/4.0/.

\section{References}

1. Marrero JA, Kulik LM, Sirlin CB, et al. Diagnosis, Staging, and Management of Hepatocellular Carcinoma: 2018 Practice Guidance by the American Association for the Study of Liver Diseases. Hepatology 2018;68:723-50.

2. Kanwal F, Befeler A, Chari RS, et al. Potentially curative treatment in patients with hepatocellular cancer--results from the liver cancer research network. Aliment Pharmacol Ther 2012;36:257-65.

3. Tabrizian P, Jibara G, Shrager B, et al. Recurrence of hepatocellular cancer after resection: patterns, treatments, and prognosis. Ann Surg 2015;261:947-55.

4. Imamura $\mathrm{H}$, Matsuyama $\mathrm{Y}$, Tanaka E, et al. Risk factors contributing to early and late phase intrahepatic recurrence of hepatocellular carcinoma after hepatectomy. J Hepatol 2003;38:200-7.

5. Poon RT, Fan ST, Ng IO, et al. Different risk factors and prognosis for early and late intrahepatic recurrence after resection of hepatocellular carcinoma. Cancer 2000;89:500-7.

6. Wu JC, Huang YH, Chau GY, et al. Risk factors for early and late recurrence in hepatitis B-related hepatocellular carcinoma. J Hepatol 2009;51:890-7.

7. Sohn W, Paik YH, Kim JM, et al. HBV DNA and HBsAg levels as risk predictors of early and late recurrence after curative resection of HBV-related hepatocellular carcinoma. Ann Surg Oncol 2014;21:2429-35.

8. Cheng Z, Yang P, Qu S, et al. Risk factors and management for early and late intrahepatic recurrence of solitary hepatocellular carcinoma after curative resection. HPB (Oxford) 2015;17:422-7.

9. Zhang X, Li J, Shen F, et al. Significance of presence of microvascular invasion in specimens obtained after surgical treatment of hepatocellular carcinoma. J Gastroenterol Hepatol 2018;33:347-54.

10. Rodríguez-Perálvarez M, Luong TV, Andreana L, et al. A systematic review of microvascular invasion in hepatocellular carcinoma: diagnostic and prognostic variability. Ann Surg Oncol 2013;20:325-39.

11. Lee S, Kang TW, Song KD, et al. Effect of Microvascular Invasion Risk on Early Recurrence of Hepatocellular Carcinoma After Surgery and Radiofrequency Ablation. Ann Surg 2021;273:564-71.

12. Bruix J, Sherman M, Practice Guidelines Committee AAftSoLD. Management of hepatocellular carcinoma. 
Hepatology 2005;42:1208-36.

13. Zhou WP, Lai EC, Li AJ, et al. A prospective, randomized, controlled trial of preoperative transarterial chemoembolization for resectable large hepatocellular carcinoma. Ann Surg 2009;249:195-202.

14. Shi M, Guo RP, Lin XJ, et al. Partial hepatectomy with wide versus narrow resection margin for solitary hepatocellular carcinoma: a prospective randomized trial. Ann Surg 2007;245:36-43.

15. Roayaie S, Blume IN, Thung SN, et al. A system of classifying microvascular invasion to predict outcome after resection in patients with hepatocellular carcinoma. Gastroenterology 2009;137:850-5.

16. Benedetto U, Head SJ, Angelini GD, et al. Statistical primer: propensity score matching and its alternatives. Eur J Cardiothorac Surg 2018;53:1112-7.

17. Rubin DB, Thomas N. Matching using estimated propensity scores: relating theory to practice. Biometrics 1996;52:249-64.

18. Yang T, Lu JH, Lau WY, et al. Perioperative blood transfusion does not influence recurrence-free and overall survivals after curative resection for hepatocellular carcinoma: A Propensity Score Matching Analysis. J Hepatol 2016;64:583-93.

19. Li Z, Lei Z, Xia Y, et al. Association of Preoperative Antiviral Treatment With Incidences of Microvascular Invasion and Early Tumor Recurrence in Hepatitis B Virus-Related Hepatocellular Carcinoma. JAMA Surg 2018;153:e182721.

20. Sumie S, Kuromatsu R, Okuda K, et al. Microvascular invasion in patients with hepatocellular carcinoma and its predictable clinicopathological factors. Ann Surg Oncol 2008;15:1375-82.

21. Lim KC, Chow PK, Allen JC, et al. Microvascular invasion is a better predictor of tumor recurrence and overall survival following surgical resection for hepatocellular carcinoma compared to the Milan criteria. Ann Surg 2011;254:108-13.

22. Xu X, Zhang HL, Liu QP, et al. Radiomic analysis of contrast-enhanced CT predicts microvascular invasion and outcome in hepatocellular carcinoma. J Hepatol 2019;70:1133-44.

23. Lei Z, Li J, Wu D, et al. Nomogram for Preoperative Estimation of Microvascular Invasion Risk in Hepatitis B Virus-Related Hepatocellular Carcinoma Within the Milan Criteria. JAMA Surg 2016;151:356-63.

24. DiNorcia J, Florman SS, Haydel B, et al. Pathologic Response to Pretransplant Locoregional Therapy is
Predictive of Patient Outcome After Liver Transplantation for Hepatocellular Carcinoma: Analysis From the US Multicenter HCC Transplant Consortium. Ann Surg 2020;271:616-24.

25. Allard MA, Sebagh M, Ruiz A, et al. Does pathological response after transarterial chemoembolization for hepatocellular carcinoma in cirrhotic patients with cirrhosis predict outcome after liver resection or transplantation? J Hepatol 2015;63:83-92.

26. Kwan SW, Fidelman N, Ma E, et al. Imaging predictors of the response to transarterial chemoembolization in patients with hepatocellular carcinoma: a radiologicalpathological correlation. Liver Transpl 2012;18:727-36.

27. Yang K, Sung PS, You YK, et al. Pathologic complete response to chemoembolization improves survival outcomes after curative surgery for hepatocellular carcinoma: predictive factors of response. HPB (Oxford) 2019;21:1718-26.

28. Higuchi T, Kikuchi M, Okazaki M. Hepatocellular carcinoma after transcatheter hepatic arterial embolization. A histopathologic study of 84 resected cases. Cancer 1994;73:2259-67.

29. Golfieri R, Cappelli A, Cucchetti A, et al. Efficacy of selective transarterial chemoembolization in inducing tumor necrosis in small $(<5 \mathrm{~cm})$ hepatocellular carcinomas. Hepatology 2011;53:1580-9.

30. Bruix J, Llovet JM, Castells A, et al. Transarterial embolization versus symptomatic treatment in patients with advanced hepatocellular carcinoma: results of a randomized, controlled trial in a single institution. Hepatology 1998;27:1578-83.

31. Nishikawa H, Arimoto A, Wakasa T, et al. Effect of transcatheter arterial chemoembolization prior to surgical resection for hepatocellular carcinoma. Int J Oncol 2013;42:151-60.

32. Sotiropoulos GC, Machairas N, Fouzas I, et al. Prediction of Microvascular Tumor Invasion in Liver Transplant Candidates with Hepatocellular Carcinoma: A Feasible Concept or a Misleading Illusion? Transplant Proc 2019;51:421-3.

33. Jia ZZ, Jiang GM, Feng YL. Serum HIF-1alpha and VEGF levels pre- and post-TACE in patients with primary liver cancer. Chin Med Sci J 2011;26:158-62.

34. Li X, Feng GS, Zheng CS, et al. Expression of plasma vascular endothelial growth factor in patients with hepatocellular carcinoma and effect of transcatheter arterial chemoembolization therapy on plasma vascular endothelial growth factor level. World J Gastroenterol 
2004;10:2878-82.

35. Shim JH, Park JW, Kim JH, et al. Association between increment of serum VEGF level and prognosis after transcatheter arterial chemoembolization in hepatocellular carcinoma patients. Cancer Sci 2008;99:2037-44.

36. Sergio A, Cristofori C, Cardin R, et al. Transcatheter arterial chemoembolization (TACE) in hepatocellular carcinoma (HCC): the role of angiogenesis and invasiveness. Am J Gastroenterol 2008;103:914-21.

37. Liu L, Ren ZG, Shen Y, et al. Influence of hepatic artery occlusion on tumor growth and metastatic potential in a human orthotopic hepatoma nude mouse model: relevance of epithelial-mesenchymal transition. Cancer Sci 2010;101:120-8.

38. Huang M, Wang L, Chen J, et al. Regulation of COX2 expression and epithelial-to-mesenchymal transition by hypoxia-inducible factor-1 alpha is associated with poor prognosis in hepatocellular carcinoma patients post TACE surgery. Int J Oncol 2016;48:2144-54.

39. Xu ZY, Ding SM, Zhou L, et al. FOXC1 contributes to microvascular invasion in primary hepatocellular carcinoma via regulating epithelial-mesenchymal transition. Int J Biol Sci 2012;8:1130-41.

40. Shim JH, Kim KM, Lee YJ, et al. Complete necrosis after transarterial chemoembolization could predict prolonged survival in patients with recurrent intrahepatic hepatocellular carcinoma after curative resection. Ann Surg Oncol 2010;17:869-77.

Cite this article as: Yang Y, Dang Z, Lu P, Qian Y, Lin K, Pan Z, Lau WY, Zhou W. Impact of pathological response after preoperative transcatheter arterial chemoembolization (TACE) on incidences of microvascular invasion and early tumor recurrence in hepatocellular carcinoma: a multicenter propensity score matching analysis. HepatoBiliary Surg Nutr 2022;11(3):386-399. doi: 10.21037/hbsn-20-700
41. Bargellini I, Vignali C, Cioni R, et al. Hepatocellular carcinoma: CT for tumor response after transarterial chemoembolization in patients exceeding Milan criteria-selection parameter for liver transplantation. Radiology 2010;255:289-300.

42. Paik KY, Kim EK. Pathologic response to preoperative transarterial chemoembolization for resectable hepatocellular carcinoma may not predict recurrence after liver resection. Hepatobiliary Pancreat Dis Int 2016;15:158-64.

43. Chapiro J, Wood LD, Lin M, et al. Radiologic-pathologic analysis of contrast-enhanced and diffusion-weighted MR imaging in patients with HCC after TACE: diagnostic accuracy of $3 \mathrm{D}$ quantitative image analysis. Radiology 2014;273:746-58.

44. Najmi Varzaneh F, Pandey A, Aliyari Ghasabeh M, et al. Prediction of post-TACE necrosis of hepatocellular carcinoma usingvolumetric enhancement on MRI and volumetric oil deposition on CT, with pathological correlation. Eur Radiol 2018;28:3032-40.

45. Yang $\mathrm{P}, \mathrm{Si}$ A, Yang J, et al. A wide-margin liver resection improves long-term outcomes for patients with $\mathrm{HBV}$ related hepatocellular carcinoma with microvascular invasion. Surgery 2019;165:721-30.

46. Guo SY, Fraser MW. Propensity Score Analysis: Statistical Methods and Applications. 1st edition. SAGE Publications, Inc., 2010. 